\title{
Association of Speculum Lubrication with Pain and Papanicolaou Test Accuracy
}

\author{
Dilek Uygur, MD, Tolga Guler, MD, Eyup Yayci, MD, Tijen Atacag, MD, \\ Cem Comunoglu, MD, and Gamze Mocan Kuzey, MD
}

Purpose: To determine the effects of lubrication of the vaginal speculum before insertion during a Papanicolaou test on perceived pain and quality of the cytology specimen.

Methods: Four hundred eligible women participating in cervical cancer screening and an early detection program were randomized with respect to lubricant gel use before speculum insertion. Perceived pain during speculum insertion and cytology results were assessed in study groups.

Results: The mean age of the patients was 45.7 years (range, 23-77 years). Pain score of the NO GEL group was significantly higher than that of the GEL group $(2.3$ and 1.6 , respectively; $P<.05)$. For postmenopausal women, lubricant gel use significantly reduced the pain scores of the participants $(P<$ .05). Cytological interpretations of the Papanicolaou test specimens were comparable among all groups.

Conclusions: During the collection of Papanicolaou test specimens, lubrication of the vaginal speculum with a small amount of K-Y Jelly (a water-soluble lubricant gel) decreases the pain associated with insertion of the vaginal speculum among postmenopausal women without obscuring the cytological interpretation of conventional or liquid-based cytology. In women of reproductive age, lubrication of the speculum with K-Y Jelly does not cause a meaningful effect with respect to perceived pain. (J Am Board Fam Med 2012;25: 798-804.)

Keywords: Lubrication, Pain, Papanicolaou Test, Surgical Instruments, Vaginal Speculum, Women's Health

Speculum insertion is one of the most disturbing and painful procedures of a gynecological examination. Because it is an intrusive procedure, maneuvers to minimize pain and provide more comfort to the patient should be encouraged. Although transvaginal ultrasound probes and the fingers of the physician performing a bimanual pelvic examination always are lubricated before insertion, similar application of lubricants to vaginal speculums generally is not advised for fear of obscuring cytological evaluation. The diameter of vaginal speculums

This article was externally peer reviewed.

Submitted 30 January 2012; revised 26 March 2012; accepted 2 April 2012.

From the Department of Obstetrics and Gynecology, Dr. Zekai Tahir Burak Women Health Research and Education Hospital, Ankara, Turkey (DU); the Department of Obstetrics and Gynecology (TG, TY, TA) and the Department of Pathology (CC, GMK), Near East University Medical School, Lefkosa, TRNC, Turkey.

Funding: none.

Conflict of interest: none declared.

Corresponding author: Tolga Guler, MD, Department of Obstetrics and Gynecology, Near East University Medical School, Mersin10, Lefkosa, Turkey (E-mail: dr.tolgaguler@yahoo.com). used during routine gynecological examinations are larger than the diameters of transvaginal ultrasound probes or the finger of the physician; therefore, it is reasonable to speculate that lubrication during speculum insertion can reduce pain and discomfort of the patient.

The Papanicolaou test is a successful screening tool against cervical cancer, and it dramatically reduces the mortality of the disease. It is cost-effective, easy to perform, and highly accepted by the target population. However, fear of pain during a vaginal examination may reduce the compliance of some women with regular screening. There is convincing evidence in the literature supporting that lubrication of the speculum does not alter the cytological interpretation of cervical cytology specimens. ${ }^{1-5}$ Moreover, a recent study suggested that topical application of anesthetic cream to the genital mucosa of postmenopausal women before a vaginal examination significantly reduces the pain associated with speculum application. ${ }^{6}$ The aims of this study were to investigate the effects of lubrication during speculum insertion on pain perceived 
by women and the quality of the Papanicolaou test specimen.

\section{Methods}

\section{Study Population}

This randomized, controlled study was conducted between May and September 2011 at the Cervical Cancer Screening Unit of the outpatient service of our Obstetrics and Gynecology Department. Women older than 18 years of age who participated in cervical cancer screening and an early detection program were evaluated for participation in the study. Approval was obtained from the human ethics committee of our university. Patients were informed about the study, and informed consent was obtained from the participants. Exclusion criteria for participation were history of pelvic or vaginal surgery, active gynecological complaints, and prior intervention for abnormal cervical cytology (biopsy or conization). Data for age, parity status, obstetric history, menopausal status, and current hormone replacement or oral contraceptive use were recorded on a data sheet. Women without menses for 12 consecutive months were defined as postmenopausal status.

\section{Randomization}

Four hundred eligible patients were randomized into 2 groups: GEL $(\mathrm{n}=200)$ and NO GEL $(\mathrm{n}=$ 200). Then, each group was randomized further into 2 subgroups $(\mathrm{n}=100$ in each): NO GEL conventional cytology (CC), NO GEL liquidbased cytology (LBC), GEL-CC, and GEL-LBC. To keep a similar number of subjects in each group, a block randomization method with blocks of 4 was used, as defined previously. ${ }^{7}$ During the creation of the allocation list, blocks were chosen randomly using computer-generated random numbers.

\section{Procedures}

All patients were examined by the same physician (T.G.) during the Papanicolaou test specimen collection. The external side of the inferior blade of a disposable, bivalved, plastic vaginal speculum was lubricated with a dime-sized amount of water-soluble lubricant (K-Y Jelly, Johnson \& Johnson, New Brunswick, NJ) for the patients who were allocated to gel use. Warm water was applied to the speculum for the other patients. The physician decided on the size of the plastic speculum used. Women and all medical staff, including the cytologist but not including the primary physician and the nurse, were blinded to gel use. Specimens were collected using a Broom-like device, as recommended by the manufacturer. Cervical cytology was performed by either CC or LBC (ThinPrep, Hologic, Inc, Marlborough, MA).

\section{Outcomes}

Evaluation of pain during insertion of the speculum was the primary outcome of this study. To assess the level of perceived pain, a numeric rating scale (from 0-10) was used. After the Papanicolaou test, patients were asked by a blinded member of the medical staff to rate their pain to eliminate possible hesitancy to report high pain scores to the primary physician. Their responses were recorded and analyzed as the pain score and compared between the GEL and NO GEL groups. Ratios of unsatisfactory cytology results, abnormal cells, and Papanicolaou test specimens lacking an endocervical/ transformation zone (EC/TZ) were assessed as secondary outcomes.

\section{Statistical Analysis}

Data were presented as mean \pm standard deviation, range, or percentage, as appropriate. Pearson $\chi^{2}$ tests and Student $t$ tests were used for the statistical analysis of qualitative and quantitative parameters, respectively. A commercially available statistical software package was used for analysis. A $\mathrm{P}$ value of 0.05 was set to test statistical significance.

\section{Results}

All the subjects in the GEL and NO GEL groups completed the study, as shown in the flow diagram of randomization presented as Figure 1. Table 1 presents the demographics of the study groups. The GEL and NO GEL groups were comparable with regard to age, menopausal status, and parity $(P>.05)$. None of the postmenopausal women were taking hormone replacement. Only 12 women were using oral contraceptives. There was no significant difference in the use of oral contraceptives between the GEL and NO GEL groups $(2.5 \%$ and $3.5 \%$, respectively; $P<.05$ ).

Figure 2 shows pain scores in the GEL and NO GEL groups. The overall pain score of the NO GEL group was significantly higher than that of the GEL group (mean, 2.3 [95\% CI, 0-8] and mean, 1.6 [95\% CI, 0-7], respectively; $P<.05$ ). 
Figure 1. Flow diagram of randomization.

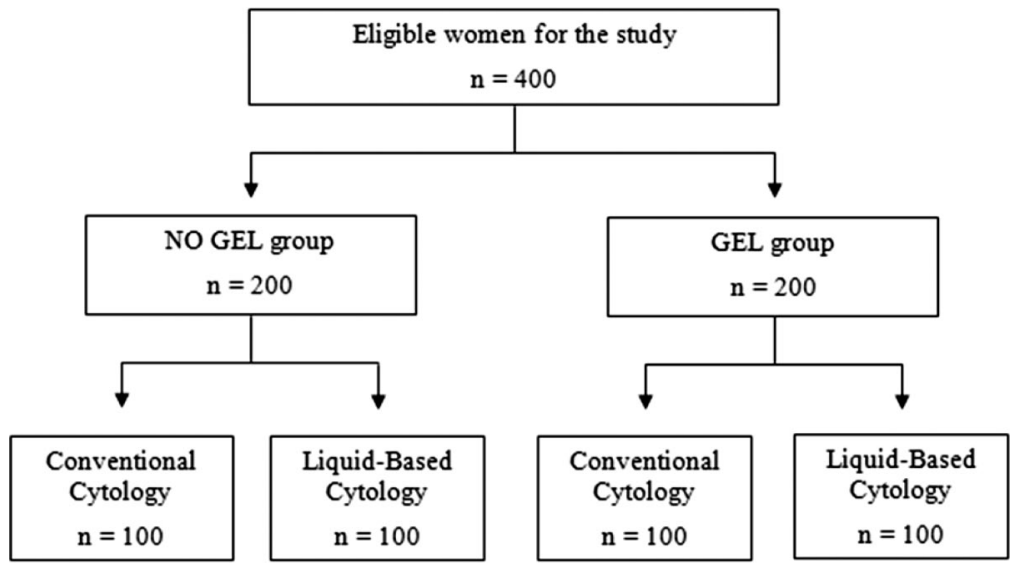

Table 2 presents the pain scores of the study groups according to menopausal status. For premenopausal women, application of the lubricant gel did not cause a meaningful change in the pain scores of the participants $(P>.05)$; however, for postmenopausal women, use of the lubricant gel significantly reduced the pain scores of the participants $(P<.05)$.

Six patients stated that this vaginal examination was their first. Their mean pain score was 4.8. Because of the small number of subjects, no statistical comparison could be made; however, the physician performing the vaginal examination personally experienced that these women were much more uncomfortable and sensible to pain when compared with others.

There were totals of 13 ASCUS, 4 low-grade squamous intraepithelial lesions (LSILs), and 1 high-grade squamous intraepithelial lesion (HSIL) reported among 400 Papanicolaou tests. No cervical cancer was reported. Papanicolaou test specimens lacking the EC/TZ component were found in

64 patients (16\%). Of the NO GEL-CC, NO GEL-LBC, GEL-CC, and GEL-LBC subgroups, ratios of Papanicolaou test specimens lacking the EC/TZ component were similar (16\%, 22\%, 12\%, and $14 \%$, respectively; $P>.05)$. The numbers of ASCUS, LSILs, and HSILs reported were too small for statistical comparison among subgroups. There were 3 unsatisfactory cytology results among 400 samples (one in each of the NO GEL-CC, GEL-CC, and GEL-LBC groups). Atrophic changes, fungal organisms, and inflammation were other main findings that were reported as findings of the Papanicolaou tests; the ratios of these findings were not statistically different between the GEL and NO GEL groups $(5.1 \%$ vs $8.0 \%, 7.1 \%$ vs $6.0 \%$, and $9.1 \%$ vs $11.6 \%$, respectively; $P>.05)$.

\section{Power Calculations}

The primary outcome of this study was to compare pain scores with respect to the use of a lubricant gel

Table 1. Demographic Characteristics of the Study Population

\begin{tabular}{lccc}
\hline Characteristics & NO GEL Group & GEL Group & \\
\hline Age (years) & & & \\
$\quad$ Mean \pm SD & $45.7 \pm 11.5$ & $44.7 \pm 12.4$ & .44 \\
$\quad$ Range & 23 to 77 & 23 to 75 & $73(36.5)$ \\
$\quad \geq 50$ (n [\%]) & $78(39)$ & $75(37.5)$ & .61 \\
Postmenopausal (n [\%]) & $79(39.5)$ & & .68 \\
Childbearing & $2 \pm 1.3$ & $1.9 \pm 1.3$ & .73 \\
Parity (mean \pm SD) & $22(11)$ & $29(14.5)$ & .29 \\
$\quad$ Nulliparity (n [\%]) & $141(70.5)$ & $132(66)$ & .33 \\
$\geq 1$ vaginal delivery (n [\%]) & & & \\
\hline
\end{tabular}

SD, standard deviation. 
Figure 2. Pain scores in the GEL and NO GEL groups. Error bars indicate standard error of mean. $* P<.05$ versus the NO GEL group.

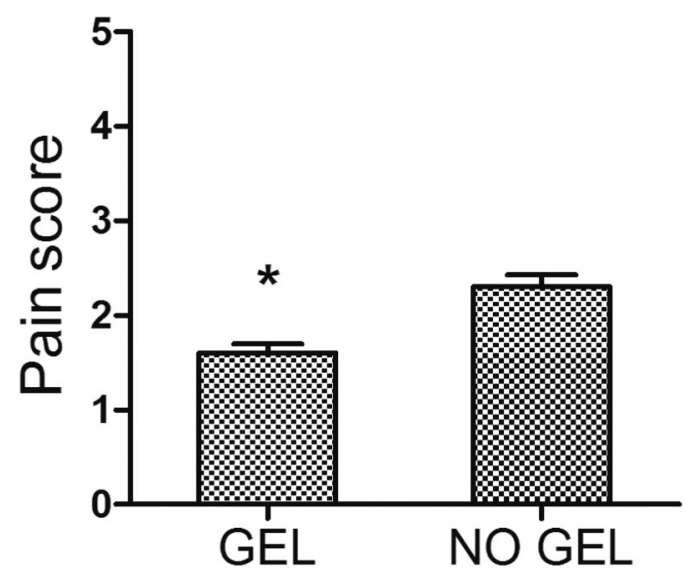

during speculum examination. Because there is no previous data representing a similar population that can be used to estimate standard deviations of the groups, a priori power calculation was not performed. Post hoc power analyses using calculated standard deviations revealed Student $t$ tests in this study with a desired power of $\geq 90 \%$ to detect a difference of 0.5 units in mean pain scores among 200 subjects in each group (2-tailed significance level $=0.05)$. This study was not stratified for menopausal status; however, power analyses of the investigation of the use of lubricant gel in the postmenopausal subgroup $(\mathrm{n}=154)$ also revealed a power of $\geq 90 \%$ to detect the significant difference in mean pain scores (2-tailed significance level $=$ $0.05)$.

\section{Discussion}

In this study, we randomized 400 women (154 of them were postmenopausal) into the GEL and NO GEL groups. In the NO GEL group, warm water was applied to the speculum before insertion. We used a horizontal numeric rating scale $(0-10$ points) for self-report of pain because it has been validated and is accepted to be universally suitable for clinical assessment of pain intensity in adults. ${ }^{8}$ There was no difference between the GEL and NO GEL groups with respect to demographic variables. In this study, overall, lubrication of the speculum with a small amount of K-Y Kelly (a watersoluble lubricant gel) during the collection of Papanicolaou test specimens decreased the pain associated with insertion of the vaginal speculum without obscuring the cytological interpretation of CC or LBC. In postmenopausal women, lubrication of the vaginal speculum with K-Y Jelly had a more pronounced effect on reducing perceived pain during the Papanicolaou test. This is an important finding because sometimes speculum application can be painful for postmenopausal patients and difficult for physicians.

During vaginal examination and gynecological tests such as Papanicolaou test, health professionals prefer using water or several types of lubricants to make digital examination and speculum insertion easy. Although speculum insertion without any lubrication can cause severe pain in some patients, lubrication of the speculum before insertion during the collection of Papanicolaou test specimens generally is not advised for fear of obscuring the cytological interpretation of the specimen. ${ }^{5}$ However, there is convincing evidence in the literature suggesting that water-based lubricants do not obscure cytology results when used in small amounts before speculum insertion. ${ }^{1-3}$ Nevertheless, there are several reports suggesting that water-based lubricants might affect the LBC specimen preparation procedure when direct contamination occurs. ${ }^{9-12} \mathrm{Al}$ though direct contamination of the liquid in the vial is not a concern in clinical practice, we cannot exclude the possibility of an indirect contamination of the specimen collected from cervix (if the lubricant sticks to cervix).

In the study by Gilson et $\mathrm{al}^{13}$ a numeric pain scale was used to investigate the discomfort level of patients. They used a dry speculum to obtain $\mathrm{Pa}$ -

Table 2. Pain Scores of the NO GEL and GEL Groups with Respect to Menopausal Status

\begin{tabular}{lcccr}
\hline Menopausal Status & NO GEL $(\mathrm{n}=200)$ & GEL $(\mathrm{n}=200)$ & Mean Difference $(95 \%$ CI $)$ & $P$ \\
\hline Premenopausal & $1.8(0-7)$ & $1.6(0-7)$ & $0.2(-0.1$ to 0.5$)$ & .25 \\
Postmenopausal & $3.2(0-8)$ & $1.7(0-6)$ & $1.5(1.0-2.1)$ & $<.05$ \\
\hline
\end{tabular}

Pain scores are mean (minimum-maximum).

CI, confidence interval. 
panicolaou test specimens in 70 patients. After the first Papanicolaou test, they randomized same patients into 2 groups: with or without a water-based lubricant gel applied to the blades of the speculum. Gibson et al found no meaningful difference between the discomfort levels of those 2 groups. They reported that both groups had slightly less discomfort during the second Papanicolaou test, but this change was found to be nonsignificant. Their study group mainly was composed of premenopausal women (only 10 women were postmenopausal).

According to our findings, in postmenopausal women, lubrication of the vaginal speculum before insertion provided a more pronounced effect on reducing perceived pain during the collection of Papanicolaou test specimens. We suggest that atrophic changes in menopause make these women more vulnerable to pain during speculum insertion. Because our study group included a relatively high number of postmenopausal women, the overall significant effect of gel application can be explained by the effectiveness in this subgroup. The reason for the nonsignificant results of Gilson et $\mathrm{al}^{13}$ in discomfort rating regarding lubricant gel use may be the low number of postmenopausal women in their study (the group included 10 postmenopausal and 60 premenopausal women).

During Papanicolaou test evaluation, a main concern of physicians and cytologists is to detect cervical cell abnormalities related to precancerous lesions. The quality of the Papanicolaou test specimen is also important for the follow-up of the screened women. Therefore, the aim of physicians should be to obtain good-quality Papanicolaou test specimens that are not affected by lubricant agents. The 2001 Bethesda System divided adequacy categories into 2 groups: "satisfactory for evaluation" and "unsatisfactory for evaluation." ${ }^{14}$ In 2002 a task force of the American Society for Colposcopy and Cervical Pathology recommended a guideline related to the adequacy of Papanicolaou test specimens. ${ }^{15}$ By definition, any specimen with abnormal cells is reported to be satisfactory for evaluation. Although the absence of an EC/TZ component is considered to be a quality indicator, this does not make a Papanicolaou test specimen unsatisfactory. Squamous intraepithelial lesion cells are suggested to be more prevalent in specimens when EC/TZ cells are present. ${ }^{16}$ Because the presence or absence of the EC/TZ component is considered to be a quality indicator, we also evaluated the ratio of
Papanicolaou test specimens lacking the EC/TZ component as one of our secondary outcomes among the 4 subgroups of the study. The other secondary outcomes were the ratios of unsatisfactory cytology results and abnormal cells. There were 13 ASCUS (3.3\%), 4 LSILs, and 1 HSIL reported in this study. Overall, three Papanicolaou test specimens (of 400 samples) were reported to be unsatisfactory (one each in the NO GEL-CC, GEL-CC, and GEL-LBC subgroups). The ratio of Papanicolaou test specimens lacking the EC/TZ component were comparable between the 4 subgroups. The use of K-Y Jelly in this study was not related to poor quality of cervical cytology. As a quality parameter, it is recommended that laboratory rates of ASCUS should be less than $5 \%$ among low-risk populations and less than 2 to 3 times the rate of squamous intraepithelial lesions in high-risk populations. ${ }^{17}$ Overall, our ASCUS rate is comparable with this recommendation, which is another quality assurance of this study.

There is no prospective study in the literature that was designed for investigating the detection rate of cervical cancer when lubricants were used during a Papanicolaou test; however, there are several reports consistent with our findings. Griffith et $\mathrm{al}^{3}$ reported that water-based lubricants do not change the ratio of unsatisfactory cytology results. They also mentioned that the use of a small amount of lubricant gel does not alter Chlamydia trachomatis diagnosis by DNA probe assay. The ratio of unsatisfactory cytology results was found to be the same for clinics that used a lubricant versus those that did not use a lubricant in the study by Amies et $\mathrm{al}^{1}$; however, there are several reports in the literature that suggested that lubricants can affect the result of cervical cytology. ${ }^{9-12,18}$ It was reported that certain lubricants containing carbopol polymers can interfere with the ThinPrep filter during processing. ${ }^{12}$ In most of these studies, lubricant gel either was added intentionally to LBC specimens or directly contaminated the cervix. Most of these study designs do not reflect actual clinical practice. However, in a recent study, Köşüş et $\mathrm{al}^{18}$ reported that the ratio of unsatisfactory Papanicolaou test results increased without a meaningful change in the rate of detection of premalignant lesions when a plastic speculum lubricated with a water-based gel was used during a Papanicolaou test. This was a retrospective cohort study and there was more than one health care professional that performed the Papa- 
nicolaou test specimen collection; these professionals had different levels of experience.

The collection of Papanicolaou test specimens should be the first component of a pelvic examination before transvaginal solography or bimanual pelvic examination because direct contamination of the cervix with a lubricant gel will certainly happen in those circumstances. This kind of contamination was shown to have an obscuring effect on cytological evaluation. ${ }^{9}$ When lubricant gel is applied to the speculum blades, contamination of the cervix with the gel cannot be excluded in some cases, but our results suggest that application of K-Y Jelly in small amounts to the outer surface of the speculum did not interfere with the quality of the Papanicolaou test specimens. We have evaluated use of lubricant gel in the collection of cervical specimens along with LBC or CC and found similar results for both. It is difficult to quantify the upper limit of the lubricant, but it can be stated that a small amount (just enough to lubricate the blades of speculum) carefully applied does not seem to obscure interpretation of either CC or LBC.

There are limited data in the literature about the experience of the first pelvic examination. Adolescents generally are anxious and embarrassed ${ }^{19}$; however, the pain or discomfort of women during their first pelvic examination was not evaluated in detail. According to the personal experience of the physician who performed the vaginal examinations in this study, these women are more prone and sensitive to pain. If the first pelvic examination is uncomfortable, some women may hesitate to participate in forthcoming screening programs. More studies are needed to investigate whether the use of lubricant gel during the first pelvic examination will improve the rate of attendance to cervical screening programs.

There are few studies investigating the effects of lubricant gel on patient comfort during speculum insertion. Our study population consisted of both premenopausal and postmenopausal women. We also collected Papanicolaou test specimens for both CC and LBC. We used a randomized design for both gel application and type of cytology. All patients were examined by the same physician during the collection of Papanicolaou test specimens; thus, an important source of variability was eliminated. These aspects of our study are important to evaluate the place of lubricant gel use in the collection of Papanicolaou test specimens.

\section{Limitations}

The limitation of our study is that we used only one type of water-based lubricant gel (K-Y Jelly); therefore, our results should not be generalized to other types lubricants. Pain perception is multidimensional and complex, and for gynecologic examinations and procedures, there may be other cultural factors that can affect these results in different populations. Further research with different types of lubricants conducted with premenopausal and menopausal women is needed to determine the place of lubrication of the vaginal speculum during Papanicolaou tests.

\section{Conclusions}

Overall, during the collection of Papanicolaou test specimens, lubrication of the speculum with a small amount of K-Y Jelly reduces the pain associated with insertion of the vaginal speculum without obscuring the cytological interpretation of $\mathrm{CC}$ or LBC. In postmenopausal women, lubrication of the speculum with K-Y Jelly causes a more pronounced effect on reducing perceived pain during Papanicolaou tests. The collection of Papanicolaou test specimens using a small amount of a water-soluble lubricant gel such as K-Y Jelly before speculum insertion may be helpful for health professionals to increase the comfort of patients by reducing procedure-related pain.

\section{References}

1. Amies AM, Miller L, Lee SK, Koutsky L. The effect of vaginal speculum lubrication on the rate of unsatisfactory cervical cytology diagnosis. Obstet Gynecol 2002;100(5 Pt 1):889-92.

2. Hathaway JK, Pathak PK, Maney R. Is liquid-based Pap testing affected by water-based lubricant? Obstet Gynecol 2006;107:66-70.

3. Griffith WF, Stuart GS, Gluck KL, Heartwell SF. Vaginal speculum lubrication and its effects on cervical cytology and microbiology. Contraception 2005;72:60-4.

4. Allan GM, Korownyk C, Ivers N. Papanicolaou tests: does lubricant reduce the quality or adequacy? Can Fam Physician 2011;57:309.

5. Harmanli O, Jones KA. Using lubricant for speculum insertion. Obstet Gynecol 2010;116(2 Pt 1): 415-7.

6. Keskin AE, Onaran Y, Duvan IC, Simavli S, Kafali H. Topical anesthetic (lidocaine-prilocaine) cream application before speculum examination in postmenopausal women. J Minim Invasive Gynecol 2012;19:350-5. 
7. Altman DG, Bland JM. How to randomise. BMJ 1999;319:703-4.

8. Dalton JA, McNaull F. A call for standardizing the clinical rating of pain intensity using a 0 to 10 rating scale. Cancer Nurs 1998;21:46-9.

9. Charoenkwan K, Ninunanahaeminda K, Khunamornpong S, Srisomboon J, Thorner PS. Effects of gel lubricant on cervical cytology. Acta Cytol 2008;52:654-8.

10. Martinez-Giron R, Martinez-Torre S, FernandezGarcia C. Vaginal lubricant gel as an artefact on a Pap smear. Diagn Cytopathol 2011;39:431.

11. Holton T, Smith D, Terry M, Madgwick A, Levine T. The effect of lubricant contamination on ThinPrep (Cytyc) cervical cytology liquid-based preparations. Cytopathology 2008;19:236-43.

12. Feit TD, Mowry DA. Interference potential of personal lubricants and vaginal medications on ThinPrep Pap tests. J Am Board Fam Med 2011;24: 181-6.

13. Gilson M, Desai A, Cardoza-Favarato G, Vroman P, Thornton JA. Does gel affect cytology or comfort in the screening Papanicolaou smear? J Am Board Fam Med 2006;19:340-4.
14. Solomon D, Davey D, Kurman R, et al. The 2001 Bethesda system: terminology for reporting results of cervical cytology. JAMA 2002;287:2114-9.

15. Davey DD, Cox JT, Austin RM, et al. Cervical cytology specimen adequacy: patient management guidelines and optimizing specimen collection. J Low Genit Tract Dis 2008;12:71-81.

16. Davey DD, Austin RM, Birdsong G, et al. ASCCP patient management guidelines: Pap test specimen adequacy and quality indicators. Am J Clin Pathol 2002;118:714-8.

17. McGrath CM. ASCUS in Papanicolaou smears. Problems, controversies, and potential future directions. Am J Clin Pathol 2002;117(Suppl):S62-75.

18. Köşüş A, Köşüş N, Duran M, Haltaş H, Hızlı D, Kafali H. Effect of liquid-based gel application during speculum examination on satisfactory level of smear examination. Arch Gynecol Obstet 2012;285: 1599-602.

19. Oscarsson MG, Benzein EG, Wijma BE. The first pelvic examination. J Psychosom Obstet Gynaecol 2007;28:7-12. 